\title{
Pregnancy Induced Ocular Changes and Associated Risk of Ocular Medications
}

\author{
Kaur G, Isac DM, Thomas S, Koshy J \\ Department of Ophthalmology, Christian Medical College, Ludhiana, India
}

Received: Jan 05, 2014; Accepted: August 14, 2014

\begin{abstract}
Pregnancy is associated with various ocular changes which can be either physiological or pathological or modification in pre existing conditions. These changes are mostly transient, however some can become permanent. Physiological ocular changes include change in ocular adnexa, tear film composition, cornea, refraction and intraocular pressure. Pathological ocular changes occur as a result of pregnancy induced hypertension, pseudotumour cerebri, vascular occlusive disorders and amniotic fluid embolism. Some of the pre-existing ocular conditions worsen during pregnancy which include diabetic retinopathy, Grave's disease, toxoplasmosis, pituitary tumours, meningioma and uveal tumours. Others show improvement in pregnancy such as uveitis, optic neuritis and multiple sclerosis. Yet others are associated with exacerbations during postpartum period as optic neuritis. Knowledge of these changes is important for both ophthalmologists and gynaecologists for proper management of these patients. Also the treatment modalities for various disorders differ between pregnant and non pregnant females. The ophthalmic medications should be used cautiously during pregnancy and lactation to avoid harmful effects in the mother and the fetus. The materials published in Pubmed, Google Scholar webpages and standard books have been used for preparing this paper.
\end{abstract}

Keywords: ocular changes; ocular medications; pregnancy.

\section{INTRODUCTION}

Pregnancy is a state characterised by manyfold changes occurring in various organs of the body. Haematological, immunological and cardiovascular systems undergo the most prominent changes. These are mainly attributed to the hormones released by placenta, maternal endocrine glands and the fetal adrenal glands. ${ }^{1}$ As with other parts of the body, eyes undergo many changes during pregnancy, which are usually transient in nature but sometimes can become permanent. Knowledge of these changes is essential for both gynaecologists and ophthalmologists. In this article, we reviewed the physiological and pathological ocular changes during pregnancy, the effect of pregnancy on pre-existing eye disorders, the ocular changes during labor and the effect of ophthalmic medications during pregnancy.

\section{METHODS}

The pregnancy induced ocular changes were assessed by reviewing the available literature. The literature published in Pubmed, Google Web pages from 1985

\section{CORRESPONDENCE}

Dr Gurvinder Kaur

Department of Ophthalmology, Christian Medical College,

Ludhiana, Punjab, India

Email: gurvinder.eye@gmail.com

Phone: +091-9872210103 to 2012 were searched for this purpose. We also looked at other relevant publications like reports, training manuals and books.

\section{THE PHYSIOLOGICAL OCULAR CHANGES}

The physiological changes in the eye during pregnancy occur at both structural and functional levels.

\section{Ocular adnexa}

Chloasma manifests as increased pigmentation around the eyes and cheeks. ${ }^{2}$ Spider angioma is a type of telangectasia which commonly develops during pregnancy on the face and upper body. ${ }^{3}$ Ptosis has been reported during and after normal pregnancy and is usually unilateral. It is attributed to the defect developing in the levator aponuerosis because of fluid retention and hormonal changes. ${ }^{4}$ All these changes resolve during postpartum period. Ocular motility defects can present for the first time during pregnancy for which pre-existing conditions such as Grave's disease need to be ruled out. ${ }^{5}$

\section{Tear composition}

Alterations in the composition of the tear film occur during pregnancy. The secretion of lysozyme, a viscous component of the tear film is increased during pregnancy. ${ }^{6}$ Disruption of lacrimal acinar cells may lead to pregnancy induced dry eye syndrome. ${ }^{7}$ These changes can lead to contact lens intolerance or greasy deposits on the contact lenses. 


\section{Conjunctival vessels}

In the third trimester, reduced visibility of conjunctival capillaries and conjunctival arteriolar spasm may occur possibly because of the gradual decrease in blood flow rate. The conjunctival veins may take on a granular appearance. These changes reverse during post partum period. ${ }^{6}$

\section{Cornea}

There is an increase in both corneal thickness and corneal curvature in the third trimester and in the post partum period. This may lead to temporary alterations in refraction. Hormone induced corneal edema may be responsible for these corneal changes. These changes revert to normal after delivery. ${ }^{89}$ Corneal sensitivity has been reported to decrease during pregnancy. These changes may aggravate contact lens intolerance during pregnancy., ${ }^{5,10}$ Krukenberg spindles can develop early in pregnancy and they tend to decrease in size during the third trimester and postpartum. This is attributed to increasing levels of progesterone and aqueous outflow during pregnancy. Progesterone is known to increase stromal melanin phagocytosis leading to increased clearance of pigment. ${ }^{11}$

\section{Refractive error and accommodation}

Transient loss of accommodation has been seen during pregnancy and lactation. ${ }^{12}$ The tendency of fluid retention during pregnancy and lactation affects refraction. The patient should be aware of possible fluctuations in vision and that glasses prescribed during this time may become unsuitable later. It is advisable that any changes in eyeglass prescriptions should be postponed until several weeks postpartum. The results of refractive eye surgery before, during or immediately after pregnancy are also unpredictable. Thus refractive surgery should be postponed until there is a stable postpartum refraction. ${ }^{13}$

\section{Aqueous circulation}

The intra-ocular pressure decreases steadily during pregnancy and continues for several months postpartum. ${ }^{13}$ Thus improvement in preexisting glaucoma has been observed during pregnancy. In ocular hypertensives, pregnancy can decrease IOP up to a level of normal limit. ${ }^{11}$ The postulated mechanisms for reduced IOP are increase in the facility of aqueous outflow because of increased levels of prostaglandins and $\beta$ - human chorionic gonadotropin, decreased episcleral venous pressure related to the generalized decrease in peripheral vascular resistance and increased tissue elasticity leading to reduced scleral rigidity. ${ }^{14}$

\section{Visual field changes}

There are conflicting reports on changes in visual field during pregnancy. Some studies have described bitemporal contractions while others have reported concentric contraction with enlargement of the blind spot. ${ }^{6}$ In some cases the changes begin early in pregnancy and progress until the time of delivery whereas in others the contraction occurs in the last month of pregnancy and remains unchanged. The proposed mechanism includes pituitary enlargement with mechanical compression of the chiasma. ${ }^{15}$

\section{THE PATHOLOGICAL OCULAR CHANGES}

There are a few pathological eye changes which are specifically related to the pregnant state of female.

\section{Pregnancy induced hypertension}

Pregnancy induced hypertension (PIH) is a systemic hypertensive disorders characterized by generalised edema and proteinuria occuring in the third trimester of pregnancy. These changes alone are termed as preeclampsia. Preeclampsia associated with seizures is termed as eclampsia. Superimposed $\mathrm{PIH}$ is the development of preeclampsia or eclampsia in a woman with preexisting systemic hypertension. It is postulated that the disorder results from generalized vasospasm related to increased sensitivity to circulating prostaglandins and angiotensin $11 .^{6}$ $\mathrm{PIH}$ has an incidence of 5\%. It usually occurs after fifth month of pregnancy but may present anytime between 3rd and 9th month of pregnancy. Retinal changes are likely to occur when diastolic BP is more than $100 \mathrm{~mm}$ of $\mathrm{Hg}$ and systolic BP is above $150 \mathrm{~mm}$ of $\mathrm{Hg}^{16}$

The most common visual symptom is blurring of vision. Other symptoms reported are photopsia, scotomas, diplopia and cortical blindness. Visual disturbances may be a precursor of a seizure in preeclamptic patients. Widespread arteriolar constriction involving vessels of conjunctiva, retina, choroid, optic nerve and occipital cortex is seen in $\mathrm{PIH}$. The most common vascular abnormality is seen in retinal arterioles, occurring in $40-100 \%$ of the $\mathrm{PIH}$ patients. Focal arteriolar narrowing is commonly seen which may progress to generalised constriction associated diffuse retinal edema, hemorrhages, exudates and cotton wool spots. ${ }^{6}$ 
Exudative retinal detachment has been reported in $1 \%$ of patients with preeclampsia and in $10 \%$ of those with eclampsia. It is usually bilateral and its pathogenesis is related to the choroidal ischemia secondary to an intense arteriolar vasospasm. ${ }^{17}$ The patients complain of a relative central scotoma in the affected eye. The majority of patients have spontaneous reattachment with complete recovery of vision with clinical management. ${ }^{6,18}$

Vascular changes in optic nerve may lead to papilledema, acute ischemic optic neuropathy and optic atrophy. Cortical blindness resulting from cerebral vasospasm is a rare complication of PIH. It is usually reversible. ${ }^{19}$

\section{Central serous chorioretinopathy}

Idiopathic central serous chorioretinopathy (CSC) is characterized by a serous retinal detachment of the sensory retina. Pregnancy is considered a risk factor for the development of central serous chorioretinopathy..$^{20}$ Patient complains of decreased vision, central scotoma, metamorphopsia and micropsia. CSC is often associated with fibrinous subretinal exudation. It usually develops in the third trimester and resolves spontaneously within 1 to 2 months after delivery with visual functions returning to normal or near-normal. The possible mechanisms include physiologic changes in hemodynamics, vascular permeability, autonomic nervous function and hormones that occur during pregnancy. ${ }^{6}$

\section{Pseudotumor cerebri}

It is characterized by increased intracranial pressure, normal cerebrospinal fluid content and normal head imaging scan. It is mostly seen in obese females of child bearing age. Pregnancy does not increase the risk of development of pseudotumour cerebri. It is mostly seen during the first and second trimester. ${ }^{21}$ It is associated with headache, visual disturbances and papilloedema. Visual field defects are the most common visual disturbances. It is a diagnosis of exclusion. Once diagnosed the decision to treat is based on visual acuity and visual field loss. Medical management is usually effective. Pregnancy is not contraindicated in women with pseudotumor cerebri, and termination of pregnancy is seldom required. ${ }^{11,22}$

\section{Vascular occlusive disorders}

A hypercoagulable state exists during pregnancy because of increased platelet adhesiveness, elevated levels of clotting factors, hyperfibrinogenemia, elevated fatty acid levels and reduced fibrinolytic activity. The resultant slowing of venous flow may predispose to thrombosis. ${ }^{6}$ Retinal artery occlusion, retinal vein occlusion, disseminated intravascular coagulopathy, amniotic fluid embolism and cerebral venous thrombosis are the vascular occlusive disorders seen during pregnancy. ${ }^{6}$

\section{Retinal artery occlusion}

Both branch and central retinal artery occlusions have been reported in pregnancy or in the immediate postpartum period. A Purtscher-like retinopathy with occlusion of multiple retinal arterioles has been described after childbirth. Because of the hypercoagulable state and elevated levels of estrogens, pregnancy itself is a risk factor for retinal artery occlusion. ${ }^{6}$

\section{Retinal vein occlusion}

Retinal vein occlusions are less common than arterial occlusions. It has been associated with impaired fibrinolysis and large fluctuations in IOP. Treatment with aspirin has been suggested and the condition resolves after delivery. ${ }^{6}$

\section{Disseminated intravascular coagulopathy}

It is a haemorrhagic syndrome resulting from activation of clotting factors and fibrinolytic enzymes which leads to widespread thrombosis and tissue necrosis. It may develop in disorders of pregnancy such as abruptio placentae, complicated abortions, intrauterine death and preeclampsia. Visual symptoms may develop and tend to be of early and sudden onset. Extensive areas of choroidal occlusion, serous retinal detachments and choroidal haemorrhages may occur. Changes tend to be bilaterally symmetrical. Both visual acuity and retinal detachment resolve after recovery from the systemic disorder. ${ }^{6,23}$

\section{Amnoitic fluid embolism}

It is serious complication of pregnancy with a high mortality rate. It results in anaphylaxis and disseminated intravascular coagulopathy. Ocular manifestations include bilateral retinal arteriolar occlusions caused by embolization of particulate matter in the amniotic fluid and retinal and choroidal ischemia following massive hemorrhage. ${ }^{6,24}$

\section{Cerebral venous thrombosis}

It is a known complication of the post partum period. It presents with severe headache and may progress 
to seizures, focal neurological deficits and visual disturbances. Visual field defects occur in $6 \%$ of cases. Cortical blindness has also been reported. ${ }^{6}$

\section{EFFECT OF PREGNANCY ON PRE-EXISTING EYE DISEASES}

Various pre-existing eye diseases show either worsening or improvement during pregnancy

\section{Uveitis}

Uveitis is an ocular disease characterised by inflammation of the uveal tract which include iris, ciliary body and choroid. Pregnancy is associated with lower numbers of flare-ups of non-infectious uveitis compared to the non pregnant state. ${ }^{25}$ The immunosuppressive state and high levels of steroids present in pregnant women may have a beneficial effect on uveitis during pregnancy but there is a risk of exacerbation of the disease in the postpartum period. $^{26}$

\section{Uveal tumors}

The occurrence of malignant melanoma of the choroid during pregnancy is rare. In contrast, cutaneous melanomas during pregnancy are frequently reported. ${ }^{6}$ Although hormonal influence is suggested for the growth of melanomas in pregnancy, recent evidence has suggested that estrogen and progesterone do not have any role in the development or progression of uveal melanomas. ${ }^{15}$

Choroidal haemangiomas undergo rapid growth during pregnancy but some regress postpartum. ${ }^{27}$

\section{Diabetic retinopathy}

Diabetic retinopathy is the leading cause of blindness between 24 to 64 years of age. Many females of child bearing age correspond to this group. It has been well documented that pregnancy can worsen pre existing diabetic retinopathy. Diabetic changes that occur during pregnancy are similar to those seen in non pregnant diabetic patients. ${ }^{28}$

Pregnancy is considered as an independent risk factor for development and progression of diabetic retinopathy especially in women with insulin dependent diabetes mellitus. Also the progression of diabetic retinopathy during pregnancy is related to the degree of retinopathy prior to conception, duration of diabetes, metabolic control prior to pregnancy, adequacy of treatment and presence of additional vascular disorders like pre-existing or concomitant hypertension. ${ }^{15}$ As the changes and progression are still strongly correlated with duration of diabetes, these females are encouraged to plan pregnancies early in life if possible. ${ }^{29}$

In Diabetes in Early Pregnancy Study (DIEP), progression of diabetic retinopathy was seen in $21.1 \%$ women with mild non proliferative diabetic retinopathy and $54.8 \%$ women with moderate to severe nonproliferative retinopathy. Also severe form of diabetic retinopathy is associated with higher rates of fetal deaths and congenital malformations. Thus in women with severe proliferative changes before conception; pregnancy should be deferred till the disease is treated and a good glycemic control is achieved. ${ }^{15,30}$

Retinopathy changes that have progressed during pregnancy have a tendency to regress after delivery. ${ }^{30}$

Gestational diabetes is diabetes mellitus that develops during pregnancy. Reports suggest that it has not been associated with the development of retinopathy and thus does not require regular eye examination during pregnancy. ${ }^{31}$

According to The American Academy of Ophthalmology recommendations, all diabetic women should have an eye examination before conception to determine the baseline severity of diabetic retinopathy. The subsequent eye examinations should be planned once in each trimester or earlier as advised by the ophthalmologist according to the severity of retinopathy. ${ }^{30}$

Laser photocoagulation should be considered for pregnant women with severe non proliferative diabetic retinopathy. The treatment should not be delayed till proliferative changes develop because proliferative diabetic retinopathy has a tendency to progress despite treatment. Regular follow-ups are required during pregnancy and in the postpartum period in this group of patients until the retinopathy has stabilized. ${ }^{32}$

Indications for surgery during pregnancy include tractional retinal detachment, non-clearing vitreous haemorrhage and neovascular glaucoma. ${ }^{15}$

Diabetic macular edema may develop or worsen during pregnancy. It is increased by coexisting hypertension, nephropathy and proteinuria. The treatment should be delayed until postpartum period as there is possibility of spontaneous recovery. Cases in whom clinically significant macular edema persists after delivery should be treated with focal laser photo- 
coagulation. $^{33,34}$

\section{Grave's disease}

Graves' disease is the most common cause of hyperthyroidism in pregnancy. It is an important cause of unilateral and bilateral proptosis. Graves' disease aggravates in the first trimester and after delivery but improves in the second and third trimester. ${ }^{6,35}$ Rarely the fetus can be affected because of transplacental passage of maternal IgG. Pregnant female with Graves's orbitopathy is treated in the same manner as in a non-pregnant female. Mothers with active Graves' disease should be treated with antithyroid drugs, because in untreated cases there is high rate of abortions, intrauterine and perinatal deaths, and premature births. The lowest possible dose should be used so as to maintain maternal free thyroxine levels at or just above the upper limit of the normal non pregnant reference range. The drug of choice in pregnancy is propylthiouracil. ${ }^{6,36}$

\section{Toxoplasmosis}

Primary infection in mother during pregnancy can result in transplacental transmission of infection to the fetus. Latent ocular toxoplasmosis may reactivate during pregnancy in the mother. Toxoplasmosis infection typically presents as retinochoroiditis. Decreased vision and floaters are the most frequent symptoms. Active infection typically presents as greywhite retinal necrosis with choroiditis, vasculitis and vitritis. The diagnosis is based on clinical findings and can be supported by the detection of antibodies and Toxoplasma gondii DNA using polymerase chain reaction (PCR) ${ }^{15}$ Women with active infection during pregnancy should be monitored for antibiotic levels once per trimester. If the titres increase, therapeutic abortion may be considered. If titres remain stable, pregnancy can be continued with small risk to the fetus. ${ }^{6,37}$

\section{Pituitary tumours}

Prolactinomas are the most common functioning tumours seen in the pregnant patients. They present a potential risk in pregnancy because pituitary gland shows physiological growth during pregnancy. ${ }^{38}$ Adenomas less than $1.0 \mathrm{~cm}$ in size are defined as micro- prolactinomas and those more than $1.0 \mathrm{~cm}$ are macro-prolactinomas. Hormone fluctuations during pregnancy can stimulate estrogen receptors on the prolactinoma which can lead to enlargement of the tumour. ${ }^{39}$ Patient presents with headache which may be followed by progressive visual field disturbances. Bi-temporal hemianopia is most common visual field defect seen. Homonymous hemi-anopia can also be seen in advanced cases. An untreated pituitary adenoma appears to increase the risk of miscarriage. ${ }^{15,40}$

Asymptomatic patients should have visual field testing every 3 months to monitor tumour growth and compression of the visual pathways. Symptomatic pituitary adenomas require the combined efforts of an ophthalmologist, obstetrician, neurosurgeon, and endocrinologist to decide upon appropriate medical, surgical, or radiation treatment. Bromocriptine, a dopamine agonist, can lower prolactin and cause shrinking of the tumor. Bromocriptine is considered safe during pregnancy. If the tumor does not respond to drugs, transsphenoidal hypophysectomy can be planned during the second trimester. ${ }^{11}$

\section{Multiple sclerosis and optic neuritis}

Patients with multiple sclerosis show stability or even improvement during pregnancy. But there is a significant increase in the risk of relapse in the postpartum period especially first three months after delivery. ${ }^{6,41}$ Puerperal immune-mediated changes are responsible for activation of optic neuritis associated with relapsing multiple sclerosis. This may result in acute loss of vision in breastfeeding women and multiple sclerosis should be considered as a cause for lactation associated loss of vision when there is no other clear cause. ${ }^{11,42}$ However studies have shown that pregnancy does not appear to exert any adverse effect on the overall course of the disease neither does multiple sclerosis has any adverse influence on fetus, pregnancy and delivery. ${ }^{6}$

\section{Meningioma}

Meningiomas are benign, slow growing tumors with male to female ratio of 1:3. Meningiomas may show a rapid growth during pregnancy that may be attributed to the presence of progesterone and estrogen receptors in tumour cells. The patient presents with a variety of visual disturbances like visual loss, visual field defects, diplopia, disc edema, optic atrophy, cranial nerve palsies and proptosis. ${ }^{43}$

Meningiomas regress postpartum but may regrow during subsequent pregnancy. Asymptomatic patients should be closely observed. The mainstay of treatment is surgical intervention. Management of meningioma in pregnancy depends on the severity 
of the symptoms and stage of gestation. In patients with mild symptoms in the last trimester, surgery is deferred until postpartum period. In cases with severe visual loss in last trimester, induction of labour and intracranial surgery is advised. If a patient comes with severe symptoms early in pregnancy, therapeutic abortion can be considered. Those who want continuation of pregnancy, steroids and hyperosmotic agents can be tried to decrease cerebral edema so that surgery can be delayed till the fetus is mature enough for delivery. ${ }^{6,33}$

\section{Ocular migraine}

There is a greater prevalence of migraine headaches among women suggesting that hormone levels especially estrogen influence the occurrence, frequency and severity of migraine attacks. There may be a change in the frequency of migraine attacks during pregnancy. Both increased and decreased frequencies have been noted. ${ }^{17}$

\section{OCULAR CHANGES DURING LABOUR}

\section{Sheehan's syndrome}

Sheehan's syndrome or pituitary apoplexy occurs as a result of ischemic pituitary necrosis due to severe postpartum haemorrhage. It may be rarely seen without massive bleeding or after normal delivery. This condition may present as a sudden onset of headache, visual loss and/or ophthalmoplegia. Enlargement of pituitary gland, small sella size, disseminated intravascular coagulation and autoimmunity have been suggested to play a role in the pathogenesis of Sheehan's syndrome in women who suffer from severe postpartum hemorrhage. ${ }^{11,44}$

\section{EFFECT OF OPHTHALMIC MEDICATIONS ON PREGNANCY}

Little is known about the risk of ophthalmic medications in pregnant and nursing women. As a general rule, the lowest possible dosage should be used. When using topical medications, nasolacrimal compression and temporary punctual occlusion could be performed to minimize systemic drug absorption. ${ }^{45}$

\section{Anti-glaucoma medications:}

Topical beta blockers (e.g. timolol) come under FDA risk category $\mathrm{C}$ in first trimester while $\mathrm{D}$ in 2 nd and $3^{\text {rd }}$ trimester. Beta blockers can cause intrauterine growth retardation if used in 2 nd and 3 rd trimester and persistent neonatal blockade if used near term.
They should be avoided during pregnancy and should be discontinued 2 to 3 days before delivery to avoid inhibiting uterine contractility. They should not be used during lactation also. ${ }^{45,46}$

Topical and systemic carbonic anhydrase inhibitors (e.g. acetazolamide, dorzolamide) are contraindicated during pregnancy because of potential teratogenic effects. They are contraindicted during lactation because of the adverse renal and hepatic effects in the infant. ${ }^{45,46}$

Prostaglandin analogs (e.g. latanoprost) fall in FDA risk category C. These are not well studied and the reports that do exist are conflicting. Their use is generally contraindicated in pregnant and lactating women. ${ }^{45,46}$

\section{Mydriatics (dilating drops)}

Use of occasional dilating drops during pregnancy for the purposes of ocular examination is safe. However, repeated use is contraindicated in pregnancy because of the potential teratogenic effects of both parasympatholytics (e.g. atropine) and sympathomimetics (e.g. phenylepinephrine). ${ }^{6,33,45}$

\section{Topical corticosteroids}

They come under FDA risk category B. Systemic corticosteroids are contraindicated in pregnancy because of teratogenic effects, stillbirths, intrauterine growth retardation and congenital cataract. Topical steroids have not been reported to have an adverse effect on pregnancy but the safety of their use has not absolutely been established. Therefore use with care and avoid their prolong use in pregnancy and lactation. ${ }^{6,33,45}$

\section{Antibiotic eye preparations}

Antibiotics which should be avoided during pregnancy are gentamycin, streptomycin, neomycin, kanamycin and fluorinated quinolones like norfloxacin and ciprofloxacin are not considered safe during pregnancy. Erythromycin and polymyxin B appears to be the safest antibiotics during pregnancy and polymyxin B and the sulfonamides are safest in lactation. ${ }^{33,45}$

\section{Antiviral eye preparations (acyclovir eye ointment)}

These fall under FDA risk category B. Topical acyclovir has not been studied in pregnant woman. However this medicine has been shown to be teratogenic in animal studies. Thus it should be used with caution in pregnancy and lactation. ${ }^{6,33,45}$ 


\section{Fluorescein dye}

It comes under FDA risk category B. No known teratogenic effects of fluorescein during pregnancy exist. In literature, there is evidence suggesting the use of fluorescein after due consideration of risk and benefit. ${ }^{47}$ Still most of the retinal specialists avoid fluorescein angiography during pregnancy, especially first trimester. Effect in lactating women is not known. ${ }^{6,33}$

\section{Topical anesthetic}

No known contraindications exist to use of topical anesthetic drops in pregnancy.

\section{Anti-allergic eye drops}

Sodium cromoglycate $2 \%$ (FDA risk category B) eye drop is safe to use in pregnancy while antihistaminic eye drops containing naphazoline (FDA category C) should be avoided. ${ }^{6,33,45}$

\section{CONCLUSIONS}

Knowledge of various ocular changes in pregnancy helps to differentiate physiological changes from pathological disorders of the eye in pregnant females, in whom treatment may differ from that in non pregnant females. Care should be taken while prescribing ocular medications to pregnant and lactating females so as to maintain the health of both mother and the baby.

\section{DISCLOSURE}

The authors report no conflicts of interest in this work. No violation of human rights and safety.

Funding: Nil

\section{REFERENCES}

12. Duke-Elder S. System of Ophthalmology. Vol.7. St. Louis: CV Mosby; 1971. p. 703

13. Dinn RB, Harris A, Marcus PS. Ocular changes in pregnancy. Obstet Gynecol Surv. 2003;58:137-44.

14. Gagne D, Pons M, Crastes de Paulet A. Analysis of the relation between receptor binding affinity and antagonist efficacy of antiglucocorticoids. J Steroid Biochem Mol Biol. 1986; 25:31522.

15. Garg P, Aggarwal P. Ocular changes in pregnancy. Nepal Ophthalmol. 2012;4(7):150-61

16. Tadin I, Bojic L, Mimca m, Karelovic D, Dogas Z. Hypertensive retinopathy and pre-eclampsia. Coll Antropol. 2001;25:77-81.

17. Prado RS, Figueiredo EL, Magalhaes TVB. Retinal detachment in Preeclampsia. Arq Bras Cardiol. 2002;79(@):185-6.

18. Prado RS, Figueiredo EL, Magalhaes TV. Retinal detachment in Preeclampsia. Arq Bras Cardiol. 2002;79:183-6.

19. Waziri-Erameh MJM, Omoti AE, Edema OT. Bilateral tota loss of vision following eclampsia- A case report. Afr J Reprod Health. 2003;7:106-8

20. Haimovici R, Koh S, Gagnon DR, Lehrfeld T, Wellik S; Central Serous Chorioretinopathy Case Control Study Group. Risk factors for central serous Chorioretinopathy- A case contro study. Ophthalmology. 2004;111:244-9.

21. Huma-Baron R, Kupersmith MT. Idiopathic intracrania hypertension in pregnancy. J Neurol. 2002;249(8):1078-81.

22. Digre KB, Varner MW, Corbett JJ. Pseudotumor cerebri and pregnancy. Neurology. 1984;34:721-9.

23. Hoines J, Buettner H. Ocular complication of disseminated intravascular coagulation (DIC) in abruption placentae. Retina. 1989;9:105-9. 
24. Chang M, Herbert WN. Retinal arteriolar occlusions following amniotic fluid Embolism. Ophthalmology. 1984;91:1634-7.

25. Kump LI, Cervantes-Castañeda RA, Androudi SN, Foster CS, Christen WG. Patterns of exacerbations of chronic non-infectious uveitis in pregnancy and Puerperium. Ocul Immunol Inflamm. 2006;14(2):99-104

26. Rabiah PK, Vitale AT. Noninfectious uveitis and pregnancy. Am J Ophthalmol. 2003;136:91-8.

27. Sunness JS, Santos A. Pregnancy and the Mother's eye. In: Duane's Clinical Ophthalmology on CD- ROM. Philadelphia: Lippincott Williams \& Wilkins; 2001.

28. Axer-Siegel R, Hod M, Fink-Cohen S, Kramer M, Weinberger D, Schindel B et al. Diabetic retinopathy during pregnancy. Ophthalmology. 1996;103:1815-9.

29. Wender-Ozegowska E, Zawiejska A, Pietryga M, Zozulinska D, Wierusz Wysocka B, Chmaj K et al. Effect of pregnancy on diabetic vascular complications. Ginekol Pol. 2004;75:342-51.

30. Mallika PS, Tan AK, Aziz S, Asok T, Syed Alwi SAR, Intan G. Diabetic retinopathy and the effect of pregnancy. Malaysian Family Physician. 2010;5(1):2-5.

31. Soubrane G, Coscas G. Influence of pregnancy on the evolution of diabetic retinopathy. Int Ophthalmol Clin. 1998;38:187-94.

32. Chan WC, Lim LT, Quinn MJ, Knox FA, McCance D, Best RM. Management and outcome of sight-threatening diabetic retinopathy in pregnancy. Eye. 2004;18:826-32.

33. Bhatia J, Sadiq MN, Chaudhary TA, Bhatia A. Eye changes and risk of ocular medications during pregnancy and their management. Pak J Ophthalmol. 2007; 23(1).

34. Preferred Practice Pattern Guidelines. Diabetic Retinopathy San Francisco, CA: American Academy of Ophthalmology. 2008:7.

35. Brown RS. Autoimmune thyroid disease in pregnant women and their offspring. Endocr Pract. 1996;2:53-61.

36. Chan GW, Mandel SJ. Therapy insight: management of Graves disease during pregnancy. Nat Clin Pract Endocrinol Metab. 2007;3:470-8
37. Pleyer U, Torun N, Liesenfeld O. Ocular toxoplasmosis. Ophthalmologe. 2007;104: 603-15

38. Frohman LA. Pituitary tumours in pregnancy. Endocrinologist. 2001;11:399-406

39. Nader S. Thyroid disease and other endocrine disorders in pregnancy. Obstet Gynecol Clin North Am. 2004;31:257-85.

40. Bronstein MD. Prolactinomas and pregnancy. Pituitary. 2005;8:31-8.

41. Bernardi S, Grasso MG, Bertollini R, Orzi F, Fieschi C. The influence of pregnancy on relapses in multiple sclerosis: a cohort study. Acta Neurol Scand. 1991;84:403-6.

42. Retzloff MG, Kobylarz EJ, Eaton C. Optic neuritis with transient total blindness during Lactation. Obstet Gynecol. 2001;98:902-4

43. Saitoh Y, Oku Y, Izumoto S, Go J. Rapid growth of meningioma during pregnancy: relationship with estrogen and progesterone receptors-case report. Neurolo Med Chir. 1989;29(5):440-3.

44. Kelestimur F. Sheehan's syndrome. Pituitary. 2003;6:181-8.

45. Chung CY, Kwok AKH, Chung KL. Use of ophthalmic medications during pregnancy. Hong Kong Med J. 2004;10(3):191-5.

46. Ho J-D, Hu C-C, Lin H-C. Antiglaucoma medications during pregnancy and risk of low birth weight: a population based study. Br J Ophthalmol.2009;93:1283-6.

47. Halperin LS, Olk RJ, Soubrane G, Coscas G. Safety of fluorescein angiography during pregnancy. Am J Ophthalmol. 1990;109(5):563-6. 